\title{
MITOCHONDRIA AS PHARMACOLOGICAL TARGETS: THE DISCOVERY OF NOVEL ANTI- OBESITY MITOCHONDRIAL UNCOUPLERS FROM AFRICA'S MEDICINAL PLANTS
}

\author{
Augustine Ocloo $^{{ }^{*}}$ and Julius Tieroyaare Dongdem ${ }^{2}$ \\ ${ }^{1}$ Department of Biochemistry, Cell and Molecular Biology, University of Ghana, P. O. Box LG54, \\ Legon, Accra, Ghana, ${ }^{2}$ Department of Medical Biochemistry, School of Medicine and Health \\ Sciences, University for Development Studies, Tamale, Ghana. \\ "E-mail: acloo@ug.edu.gh, augustineocloo@yahoo.co.uk
}

\begin{abstract}
Obesity results from prolonged positive imbalance between energy in take and expenditure. When food intake chronically exceeds the body's energy need, an efficient metabolism results in the storage of the excess energy as fat. Mitochondria are the main centre for energy production in eukaryotic cells. Mitochondrial proton cycling is responsible for a significant proportion of basal or standard metabolic rate, therefore, further uncoupling of mitochondria may be a good way to increase energy expenditure and hence represent a good pharmacological target for the treatment of obesity. This implies that, any chemical agent or photochemical compound that further uncouples the mitochondria in vivo without having any effect on mitochondria activity could be a potential target in finding treatment for obesity. In the past, uncoupling by 2, 4dinitrophenol has been used this way with notable success. This paper discusses the mitochondria as targets in the discovery of potential plant natural anti-obesity products from Africa's rich rainforests.
\end{abstract}

Key words: Uncoupling, Mitochondria, Pharmacology, Medicinal Plants, Obesity.

\section{Obesity: The Disease, Control and Treatment}

Obesity is a metabolic condition resulting from prolonged positive imbalance between energy intake and expenditure leading to the storage of excess calories as fat. An individual is said to be obese when that person is too fat for his or her well-being (Prentice, 2005; Puhl and Brownell, 2001). Technically, obesity is where the Body Mass Index (BMI) is above $30 \mathrm{~kg} / \mathrm{m}^{2}$. This condition, which was once thought to be a problem confronting developed countries, is fast becoming a global issue and a major public health problem in developing countries. In the latter, the situation is even more complicated by the co-existence of obesity with malnutrition making it extremely difficult to control (Prentice, 2006).

The rapidly increasing worldwide incidence of obesity and its association with serious comorbid diseases such as type II diabetes means it is beginning to replace other nutrition and infectious diseases as the most significant contributor to ill health (Kopelman, 2000). As a matter of fact, participants at the first International Association for the Study of Obesity (IASO) meeting in Sun City, South Africa cited obesity to be becoming epidemic in the developing world. According to them, obesity is now a major disease in Africa, along with HIV - AIDS and malnutrition and that in South Africa, one in three men and more than one in two adult women are overweight and obese, while in Morocco $40 \%$ of the population are overweight. Cultural and societal factors coupled with lack of exercise and unhealthy diets explain the rising prevalence. A call was made for urgent action to combat the epidemic in order to prevent chronic diseases linked to obesity, which ultimately slows down economic development in developing countries.

Weight loss, induced by dieting, has been shown to be successful in reducing the health consequences of obesity but unfortunately more than $90 \%$ of individuals who lose weight through dietary control eventually return to their original weight (Waden, 1993). Pharmacological treatment may therefore be desirable for patients with associated comorbid conditions who have been unable to control their obesity through diet and exercise. Any treatment for obesity has to reduce energy intake, increase energy expenditure or combine both effects. Current therapies for obesity predominantly lead to decreased energy intake either by acting at satiety centres in the brain (e.g. sibutramine) (Bray et al., 1999; James et al., 2000; Ryan, 2000), by reducing the efficiency of intestinal absorption (e.g. orlistat) (Sjostrom et al., 1998; Hvizdos and Markham, 1999) or by reducing appetite (e. g. rimonabant).

Exercise is the most practical and most potentially easiest way to increase energy expenditure. Studies have shown a sevenfold increased risk of the incidence of overweight in those with a physical activity ratio (total energy expenditure: resting metabolic rate) of $<1.8$ (Ferro-Luzzi and Martino, 1996). The main benefit of exercise is to increase metabolic rate, and overall energy expenditure, by a greater amount than that resulting directly from exercise (Poehlamn, 1989). Pharmacological agents that increase metabolic rate are likely to mimic this beneficial effect of exercise on resting metabolic rate and could provide a useful adjunct to agents acting to reduce satiety; this review will discuss how targeting mitochondrial uncoupling will help discover such potential plant natural products from Africa's rich rainforests.

\section{Uncoupling of mitochondria as a viable target for the development of anti-obesity drugs}

Mitochondria are normally responsible for $90 \%$ of cellular oxygen consumption and the majority of adenosine 
triphosphate (ATP) production (Kennedy and Lehninger, 1949). According to the chemiosmotic theory, the flow of electrons from reduced substrate to oxygen is coupled by a proton electrochemical gradient across the mitochondrial inner membrane to the synthesis of ATP from adenosine diphosphate (ADP) and phosphate (Mitchell, 1961). This process of oxidative phosphorylation can be subdivided into two distinct parts: the generation of the proton electrochemical gradient by the respiratory chain and the synthesis of ATP by the $\mathrm{F}_{0}-\mathrm{F}_{1}$ ATP synthase using the potential energy stored in the gradient (Harper et al., 2001). Naturally, not all the protons will find their way through the ATPase: some will go via other available pathways and will therefore not be used for ATP synthesis. This means that, not all of the available energy is coupled to ATP synthesis; instead, much is by uncoupled reactions when protons move from the cytosol back into the mitochondrial matrix via pathways which circumvent the ATP synthase and the other uses of the electrochemical gradient.

The importance of mitochondrial uncoupling as a potential target for finding therapeutic treatment for obesity has been extensively reviewed elsewhere (Brand, 2005; Harper et al., 2001). By simple deduction, when food intake chronically exceeds the body's energy need, an efficient metabolism results in the storage of the excess energy as fat consequently leading to weight gain and obesity. Therefore decreasing metabolic efficiency by increasing mitochondrial uncoupling will be an effective way to increase basal metabolic rate and can lead to a substantial reduction in body weight by loss of fat deposits. This uncoupling of mitochondria represents a particularly attractive target and there is already excellent proofs of the concept for this approach in humans using 2,4-dinitrophenol (DNP) (Parascandola, 1974) and in animals using adrenoceptor agonists (Weyer et al., 1999), over expression of UCP1 (uncoupling protein 1) (Li et al.. 2000) or UCP3 (Clapham et al., 2000).

Consequently, using pharmacological agents to uncouple all mitochondria throughout the body may be a high-risk treatment, because it might compromise energy homeostasis in tissues such as heart and brain. On the other hand, active tissues like these may be less susceptible to mild uncoupling than less active ones like resting muscle or resting BAT because proton conductance has much less control over respiration rate in active mitochondria (Hafner, et al., 1990). The small difference between the effective and the fatal doses of DNP, as well as side-effects resulting from its non-selective actions (Parascandola, 1974), implies that it is not in itself a suitable anti-obesity drug. Tissue selectivity and safety need to be improved for full effectiveness.

The UCP3-overexpressing mouse shows that selective uncoupling of muscle mitochondria is sufficient for a strong antiobesity effect. Specific uncoupling of brown adipose tissue mitochondria through UCP3 or in some other way remains a viable and attractive target for the development of drugs for the treatment of obesity.

Skeletal muscle represents a particularly attractive target for directed uncoupling due to the large muscle mass, which accounts for approximately $15-20 \%$ of standard metabolic rate (Rolfe and Brand, 1996; Rolfe et al., 1999). Doubling metabolic rate by modestly uncoupling skeletal muscle should produce few adverse side effects as this increase would only be equivalent to mild exercise (actually equivalent to approximately the difference between lying down and standing up (Blaxter, 1989). Indeed support for this view has been obtained using proteins that may naturally uncouple mitochondria (uncoupling proteins 1 and 3). High expression of human UCP3 in mouse skeletal muscle led to decreased weight gain despite increased food intake (Clapham et al., 2000), and expression of UCP1 in mouse skeletal muscle led to improvements in insulin sensitivity and resistance to obesity on a high fat diet (Li et al., 2000).

\section{Mitochondrial Uncouplers}

As illustrated above, the discovery of natural mitochondrial uncouplers with wide therapeutic window and high tissue specificity for the treatment of obesity has become a major challenge preoccupying most research and pharmaceutical institutions. Naturally, mitochondria have long been known to be sensitive to many compounds and are also known to play important role in drug metabolism and are thus important targets for drug development (Manash et al., 2006). Mitochondrial uncouplers are compounds that uncouple mitochondrial substrate oxidation from ATP synthesis. By so doing, they increase substrate oxidation without the corresponding increase in ATP production (Nicholls, 1974a,b). Therefore, isolated mitochondria are said to be uncoupled, when there is oxygen consumption in absence of ATP synthesis either in presence of inhibitor of ATPase (oligomycin) or in absence of ADP that is state 4 respiration as opposed to state 3 respiration which is mitochondrial respiration in presence of ADP. Uncouplers work by transporting protons across the mitochondrial inner membrane short-circuiting the normal pathway of oxidative ATP synthesis driven by proton flow and causing the loss of calories as heat.

There are two sorts of uncoupling: Basal uncoupling is not acutely regulated and is present in all mitochondria and mainly a characteristic of the bilayer, whereas inducible proton conductance is catalysed by proteins, tightly regulated and found in discrete cell types. This suggest that uncouplers could be lipophilic weak acids that picks up protons from the cytosol, diffuse across the mitochondrial inner membrane into the matrix, deprotonate and then exit as anions before repeating the catalytic cycle; or activators of specific proteins with uncoupling action such as the uncoupling proteins and the adenine nucleotide translocase. Several of the lipophilic weak acid uncouplers are known. The most notable among them are DNP, cabonyl cyanide p-trifluoromethoxyphenylhydrazone, FCCP, carbonyl cyanide m-chlorophenylhydrazone, CCCP (Harper, 1965; Lou et al., 2007). However, very few of the activators of the protein catalysed pathway have been identified. They are fatty acids, which activate proton cycling through the uncoupling proteins, superoxides and 4-hydroxynonenal (HNE), which activate the UCPs and ANT.

\section{Plants as source of drugs}

The importance of medicinal plants in the discovery of novel pharmaceuticals cannot be overemphasised and has been reviewed elsewhere (Lewis and Elvin-Lewis; 1995 Rates, 2001). The use of natural products with therapeutic properties is as ancient as human civilisation and, for a long time, mineral, plant and animal products were the main sources of drugs (see historical review by De Pasquale, 1984). Of the 252 drugs considered as basic and essential by the World Health 
Organisation (WHO), 11\% are exclusively of plant origin and a significant number are synthetic drugs obtained from natural precursors (Rates, 2001). According to Taylor, (2005), there are at least 120 distinct chemical substances derived from plants that are currently in use as important drugs in one or more countries in the world. In deed "nature has provided us with plants for food and medicine". Natural compounds can be lead compounds, allowing the design and rational planning of new drugs, biomimetic synthesis development and the discovery of new therapeutic properties not yet attributed to known compounds (Hamburger and Hostettmann, 1991).

In the process of targeting plants for new therapeutics, evaluation of collected plants is one of the important steps (Lewis and Elvin-Lewis, 1995). This includes biodirected assays which are normally followed by chemical fractionations and characterisation. Therefore the study of the effects of extracts from Africa's medicinal plants on mitochondrial substrate oxidation and membrane potential is essential to the discovery of novel anti-obesity mitochondrial uncouplers from Africa's medicinal plants.

\section{Probing Africa's Medicinal Plants}

That there are novel natural uncouplers in Africa's rich flora cannot be an overstatement. The onus is on Africa scientists in general and African Medicinal Plant Researchers in particular to conduct thorough research into identifying and characterising such compounds. Mitochondrial uncouplers cause increased oxygen consumption at same membrane potential resulting in a shift to the right of the proton leak curve providing a simple and less tedious diagnostic tool for identifying potential mitochondrial uncoupler. This simple procedure was recently explored by Lou et al. (2007), which led them to identifying uncouplers with extraordinary dynamic range. Therefore, studying the proton leak kinetics in different tissues in presence and absence of targeted plant extracts could provide a lead to discovering these important compounds.

\section{Concluding Remarks}

Mitochondria have long been known to be sensitive to many compounds and are also known to play important role in drug metabolism and are thus important targets for drug development. Mitochondrial uncouplers are potential anti-obesity agents. The search for these natural mitochondrial uncouplers has become a big challenge and of immense importance to biochemists and pharmacologists. Plants have always had the advantage of being cheap and important sources of drugs. Targeting mitochondrial uncoupling could produce a lead in discovering novel plant uncouplers with skeletal muscle specific action which could be developed into potent anti-obesity drug.

\section{Acknowledgements}

The authors are grateful to the Office of Research and Innovation Development, University of Ghana for financial support from the University of Ghana Research Grant.

\section{References}

1. Blaxter K (1989). Energy Metabolism in Animals and Man. Cambridge University Press.

2. Brand MD (1990). The contribution of the leak of protons across the mitochondrial inner membrane to standard metabolic rate. J. Theor. Biol. 145: 267-286.

3. Brand MD (2005). The efficiency and plasticity of the mitochondrial energy transduction. Biochem. Soc. 33: 897- 904.

4. Bray GA, Backburn GL, Ferguson JM, Greenway FL, Jain AK, Mendel CM, Mendels J, Ryan DH, Schwartz SL, Scheinbaum ML, Seaton TB (1999) Sibutramine produces dose-related weight loss. Obes. Res. 7: 189-198.

5. Clapham JC, Arch JR, Clapham H, Haynes A, Lister C, Moore GB, Piercy V, Carter SA, Lehner I, Smith SA, Beeley LJ, Godden RJ, Herrity N, Skehel M, Changani KK, Hockings PD, Reid DG, Squires SM, Hatcher J, Trial B, Latchman J, Rastan S, Harper AJ, Cadenas S, Buckingham JA, Brand MD, Abuin A (2000). Mice overexpressing human uncoupling protein-3 in skeletal muscle are hyperphagic and lean. Nature 406: 415-418.

6. De Pasquale A (1984). Pharmacognosy: the oldest modern science. J. Ethnopharm. 11: 1-16.

7. Ferro-Luzzi A, Martino L (1996). The origins and consequences of obesity, Wiley, Chichester.

8. Hafner RP, Brown GC, Brand MD (1990). Thyroid-hormone control of state-3 respiration in isolated rat liver mitochondria. Biochem. J. 265:731-734.

9. Hamburger M, Hostettmann K (1991). Bioactivity in plants: the link between phytochemistry and medicine. Phytochemistry 30(12): 3864-3874.

10. Harper JA, Dickinson K, Brand MD (2001). Mitochondrial uncoupling as a target for drug development for the treatment of obesity. Obes. Rev. 2: 255-265.

11. Harper VH (1965). Uncouplers of rat liver mitochondrial oxidative phosphorylation. Biochem. J. 97: 658-662.

12. Hvizdos KM, Markham, A (1999) Orlistat: a review of its use in the management of obesity. Drugs 58: 743-760.

13. James WP, Astrup A, Finer N, Hilsted J, Kopelman P, Rossner S, Saris WH, Van Gaal LF (2000). Effect of sibutramine on weight maintenance after weight loss: a randomised trial. STORM Study Group. Sibutramine Trial of Obesity Reduction and Maintenance. Lancet. 356: 2119-2125.

14. Kennedy E P, Lehninger A L (1949). Oxidation of fatty acids and tricarboxylic acid cycle intermediates by isolated rat liver mitochondria. J. Biol. Chem. 179: 957-972.

15. Kopelman PG (2000). Obesity as a medical problem. Nature 404: 635-643 
15. Lewis WH, Elvin-Lewis MP (1995). Medicinal Plants as Sources of New Therapeutics, Ann. Missouri Bot. Gard. 82: 16-24.

16. Li B, Nolte LA, Ju JS, Han DH, Coleman T, Holloszy JO, Semenkovich CF (2000). Skeletal muscle uncoupling prevents diet-induced obesity and insulin resistance in mice. Nat. Med. 6: 1115-1120.

17. Lou P, Hansen BS, Olsen PH, Tullin S, Murphy MP, Brand MD (2007). Mitochondrial uncouplers with extraordinary dynamic range. Biochem. J. 407: 129-140

18. Manash KP, Tripathy RK, Mukhopadhyay R, Mukhopadhyay AK (2006). Mitochondria - Role in different diseases: potential for drug development. CRIPS. 7: 42-46.

19. Mitchell P (1961). Uncoupling of phosphorylation to electron and hydrogen transfer by a chemiosmotic type of mechanism. Nature 191: 144-148.

20. Nicholls DG (1974a). Hamster brown-adipose-tissue mitochondria. The control of respiration and the proton electrochemical potential gradient by possible physiological effectors of the proton conductance of the inner membrane. Eur. J. Biochem. 49: 573-583.

21. Nicholls DG (1974b). The influence of respiration and ATP hydrolysis on the proton-electrochemical gradient across the inner membrane of rat-liver mitochondria as determined by ion distribution. Eur. J. Biochem. 50: 305-315.

21. Parascandola J (1974). Dinitrophenol and bioenergetics: a historical perspective. Mol. Cell Biochem. 5: 69-77.

22. Poehlman ET (1989). A review: exercise and its influence on resting energy metabolism in man. Med. Sci. Sports Exerc. 21: 515-525.

23. Prentice, AM (2005). The emerging epidemic of obesity in developing countries. Int. J. Epidemiol. 35: 93-99.

24. Puhl R. and Brownell KD (2001). Bias. Discrimination, and Obesity. Obs. Res. 9: 788 - 805.

25. Rolfe DF, Brand MD (1996). Contribution of mitochondrial proton leak to skeletal muscle respiration and to standard metabolic rate. Am. J. Physiol. 271, C1380-C1389.

26. Rolfe DF, Newman JM, Buckingham JA, Clark MG, Brand MD (1999). Contribution of mitochondrial proton leak to respiration rate in working skeletal muscle and liver and to SMR. Am. J. Physiol. 276, C692-C699.

26. Ryan DH (2000). Use of sibutramine and other noradrenergic and serotonergic drugs in the management of obesity. Endocrine 13: 193-199.

27. Rates SMK (2001). Plants as source of drugs. Toxicon. 39: 603-613.

28. Sjostrom L, Rissanen A, Andersen T, Boldrin M, Golay A, Koppeschaar HP, Krempf M (1998). Randomised placebocontrolled trial of orlistat for weight loss and prevention of weight regain obese patients. European Multicentre Orlistat Study Group. Lancet. 352: 167-172.

29. Taylor L (2005). The healing powers of rainforest herbs. pp. Square one publishers Inc. New York.

30. Waden TA (1993). Treatment of obesity by moderate and severe caloric restriction: Results of clinical research trials. Ann. Intern. Med. 119: 688-693

31. Weyer C, Gautier JF, Danforth E Jr (1999). Development of $\beta_{3}$-adrenoceptor agonists for the treatment of obesity and diabetes-an update. Diabetes Metab. 25: 11-21. 